\title{
SENSIBILITÀ DEI LIEVITI AGLI ANTIFUNGINI: VALUTAZIONE DI UN METODO DI SAGGIO ED EPIDEMIOLOGIA IN PZ IMMUNOCOMPROMESSI.
}

Basaglia G., Pancino A., Stocco Calzavara S., Sperandio P., Tomasini M.L., De Paoli P.

S.C. Microbiologia, Immunologia e Virologia, CRO,

Istituto Nazionale Tumori-IRCCS, Aviano (Pordenone).

Introduzione: I test di sensibilità in vitro dei lieviti agli antimicotici sono una metodologia che sembra destinata ad acquisire una rilevanza sempre maggiore. Data però una 
certa laboriosità e la necessità di esperienza nell'esecuzione di questi test, spesso costosi, è ragio-nevole sconsigliarne un uso routinario. Può essere invece utile il loro utilizzo in patologie particolari o in pazienti critici, in Laboratori che abbiano esperienza nell'allestimento e nell'interpretazione di questi test. Scopi del nostro lavoro sono stati:

A- Allestimento e valutazione di un test per la determinazione della sensibilità dei lieviti agli antifungini (E-test) e B- Analisi dei profili locali di sensibilità in una casistica di pazienti immunocompromessi.

Metodi:

E' stata scelta la metodica E-test (AB Biodisk, Biolife); gli antimicotici saggiati sono stati Amfotericina B, 5Fluorocitosina, Fluconazolo, Itraconazolo e Voriconazolo; i funghi studiati sono 50 ceppi di lieviti isolati da pazienti oncologici, ematologici e con AIDS, spesso immunocompromessi (C. albicans 16, C. parapsilosis 12, C. glabrata 5, C. dubliniensis 3, C. guilliermondii 2, C. tropicalis 1, C. krusei 1, C. neoformans 10).

Risultati:

A- E-test è una metodica non eccessivamente complessa, con buona riproducibilità e con buoni risultati dei controlli di qualità; necessita tuttavia, per alcuni aspetti specifici, di esperienza nell'allestimento e soprattutto nell'interpretazione (microcolonie, macrocolonie, effetto "trailing", doppi aloni, asimmetrie dell'ellisse), presenta risultati di non facile interpretazione con alcune specie (p.e. C. neoformans) e con alcuni antimicotici (p.e. alcuni azoli), ha un certo costo.

B- C. parapsilosis, C. dubliniensis e C. albicans sono risultate sensibili a tutti gli antimicotici saggiati. Resistenze sono state riscontrate per 5-fluorocitosina (C. krusei e C. neoformans), Fluconazolo (C. krusei e il $30 \%$ di C. neoformans) e Itraconazolo (C. glabrata, $C$. gugliermondi, $C$. krusei e $C$. tropicalis). Tutti i ceppi di Candida e di C. neoformans sono sensibili ad Amfotericina B e a Voriconazolo.

\section{Conclusioni:}

A- E-test, tenendone presenti pregi e limiti, è, nella nostra esperienza, una delle metodiche sicuramente utilizzabili per l'esecuzione dei test di sensibilità in vitro dei lieviti agli antimicotici.

B- Il fenomeno della resistenza nella nostra casistica è abbastanza contenuto (in totale 24 casi di resistenza su 250 test eseguiti), però presente. La presenza di resistenze, evidenziate anche nella nostra casistica, evidenziano ulteriormente da un lato l'importanza di una terapia antifungina appropriata e dall'altro l'utilità dei test di sensibilità agli antimicotici perché tale terapia sia sempre più mirata ed efficace. 